DOI: 10.5212/Publ.Biologicas.v.20i2.0003

\title{
PERFIL NUTRICIONAL E CONSUMO ALIMENTAR DE PACIENTES COM EXCESSO DE PESO ATENDIDOS POR UM AMBULATÓRIO DE NUTRIÇÃO
}

\section{NUTRITIONAL STATUS AND FOOD CONSUMPTION OF OVERWEIGHT PATIENTS TREATED BY OUTPATIENT NUTRITION}

\author{
Drielly Rodrigues VIUDES ${ }^{1 *}$, Marcela Komechen BRECAILO ${ }^{2}$, Jackeline Schwab \\ SILVA $^{1}$, Lovaine Caldas LEVINSKE ${ }^{1}$, Angelica Rocha de Freitas MELHEM ${ }^{2}$, Adriana \\ Masiero KÜHL ${ }^{2 \#}$ \\ ${ }^{1}$ Nutricionista graduada pela Universidade Estadual do Centro-Oeste (UNICENTRO). Rua: \\ Simeão Camargo Varela de Sá, 03 Bairro: Vila Carli, CEP:85.040-080. Guarapuava (PR). \\ *Autor para contato: e-mail: drika_nutri07@hotmail.com \\ ${ }^{2}$ Professora Mestre do Departamento de Nutrição da Universidade Estadual do Centro-Oeste \\ (UNICENTRO). Rua: Simeão Camargo Varela de Sá, 03 Bairro: Vila Carli, CEP:85.040- \\ 080. Guarapuava (PR)
}

\#Autores para contato: e-mail: adrimasiero@hotmail.com; angerocha@gmail.com

Data de recebimento: 10/01/2014

Data da aprovação: 05/06/2014

\section{RESUMO}

Nas últimas décadas, mudanças negativas nos padrões de atividade física e no comportamento alimentar têm provocado alterações significativas nos índices de morbimortalidade das sociedades modernas. Obesidade é uma doença crônica, multifatorial, definida como excesso de gordura corporal. Sua etiologia pode estar relacionada a: ingestão alimentar excessiva e pouco saudável, sedentarismo, fatores genéticos, metabólicos, socioculturais e psicossociais, sendo descrita como um importante problema de saúde pública da atualidade, relacionado com o desenvolvimento de diversas doenças secundárias. Objetivou-se verificar o perfil nutricional e consumo alimentar de pacientes adultos, de ambos os gêneros, com excesso de peso, atendidos pelo Ambulatório de Nutrição da Universidade Estadual do Centro-Oeste (UNICENTRO), no município de Guarapuava, PR. Trata-se de um estudo retrospectivo de análise de 22 prontuários do Ambulatório de Nutrição da UNICENTRO, considerando-se: perda de peso, inquéritos alimentares, nível socioeconômico, adesão ao tratamento nutricional e história de ganho de peso. Encontrou-se falta de adesão ao tratamento, podendo implicar modificações metabólicas que prejudicam o processo de emagrecimento e a saúde do indivíduo, por baixo consumo de alguns micronutrientes e energia. Contudo, houve melhora na distribuição percentual dos macronutrientes, principalmente de lipídios, mostrando que mesmo uma redução mínima de peso pode conseguir controlar e/ou reduzir os efeitos das doenças associadas à obesidade. Além disso, destaca-se a importância da educação nutricional.

Palavras-chave: Obesidade. Sobrepeso. Dieta. Hábitos alimentares. Adulto.

\section{ABSTRACT}

In recent decades, negative changes in patterns of physical activity and dietary behavior have caused significant changes in the pattern of morbidity and mortality in modern societies. Obesity is a chronic disease, multifactorial, defined as excess body fat. Its 
etiology may be related to overeating and unhealthy, sedentary lifestyle, genetic, metabolic, psychosocial and sociocultural, being described as a major public health problem today, being related to the development of various secondary diseases. This study assessed the nutritional status and food intake in adult patients of both genders, overweight, attended the Nutrition Clinic of the Universidade Estadual do Centro-Oeste (UNICENTRO), in Guarapuava (PR). This is a retrospective analysis of 22 medical records from the UNICENTRO's Ambulatory of Nutrition, considering weight loss, dietary, socioeconomic status, treatment adherence and nutritional history of weight gain. It was found non-adherence to treatment may result in metabolic changes that impair the process of weight loss and health of the individual, low consumption of energy and some micronutrients. However, there was improvement in the distribution of macronutrients, particularly lipids, showing that even a minimal reduction in weight may be able to control and / or reduce the effects of diseases associated with obesity. In addition, we highlight the importance of nutrition education.

Keywords: Obesity. Overweight. Diet. Food habits. Adult.

\section{Introdução}

Nas últimas décadas, mudanças nos padrões de atividade física e no comportamento alimentar provocaram alterações significativas nos índices de morbimortalidade das sociedades modernas. $\mathrm{O}$ aumento da expectativa de vida, a redução de mortes por doenças infecto-parasitárias e o aumento da mortalidade por doenças crônicas não transmissíveis (DCNTs) tornaram complexo o quadro de saúde das populações, ainda mais pelo excesso de peso como coadjuvante. (MONTEIRO, 2000).

Obesidade é uma doença crônica, multifatorial, definida como excesso de gordura corporal. Sua etiologia pode estar relacionada a: ingestão alimentar excessiva e rica em junk food, sedentarismo, fatores genéticos, metabólicos, socioculturais e psicossociais, sendo descrita como importante problema de saúde pública da atualidade, que vem ganhando destaque no cenário epidemiológico mundial. (WORLD HEALTH ORGANIZATION, 2004).

Observa-se que indivíduos com sobrepeso e obesidade apresentam maior predisposição para desenvolver hipertensão arterial sistêmica (HAS), doenças cardiovasculares (DC), diabetes mellitus (DM), dislipidemias, doenças gastrintestinais, osteoartrites, apneia do sono, distúrbios psicossociais, entre outras enfermidades secundárias ao excesso de peso, e até mesmo diminuição da expectativa de vida. (FRANCISCHI et al., 2000; SOUZA et al., 2003; FONTAINE et al., 2003).
Como citado, são vários os fatores relacionados à etiologia da obesidade, sendo, ainda, a dieta hipercalórica, devido ao consumo exacerbado de carboidratos refinados e gorduras, associada ao sedentarismo os principais fatores relacionados à elevada morbidade nos últimos vinte anos. (GUIMARÃES et al., 2010).

Estudos revelam que a perda de peso, mesmo que seja pequena, de $5 \%$ a $10 \%$ do peso inicial, é capaz de melhorar substancialmente a saúde de pacientes obesos, reduzindo fatores de risco para doenças cardiovasculares, além de melhorar o controle da HAS e DM. (FEIGEBAUM et al., 2005; AVENELL et al., 2004). Lindström et al. (2004) realizaram uma intervenção intensiva no estilo de vida de obesos durante um ano, verificando diversas mudanças benéficas na dieta, atividade física, glicose sanguínea e concentração de lipídios, além de ter obtido como resultado uma grande redução na incidência de DM. Estas alterações nas características clínicas dos obesos ocorreram independentemente da modesta redução de peso observada.

Apesar das diversas formas de tratamento, a recomendação de uma alimentação saudável e equilibrada em macro e micronutrientes para prover substratos necessários à sobrevivência e à manutenção da saúde, aliada à prática regular de atividade física, é a constituição básica de combate à obesidade. (PRICE, 2005). Contudo, estratégias para perda ponderal e para manutenção dessa perda envolvem mudanças de 


\section{PERFIL NUTRICIONAL E CONSUMO ALIMENTAR DE PACIENTES COM EXCESSO DE PESO ATENDIDOS 117 POR UM AMBULATÓRIO DE NUTRIÇÃO}

comportamento, e os resultados dependem da adesão do paciente às formas de tratamento. A prevenção e o tratamento das DCNTs, como a obesidade, em geral, exigem mudanças no estilo de vida, e na maioria dos casos observa-se pequena adesão dos pacientes a essas modificações. (ASSIS e NAHAS, 1999; HARPER e PETERSON, 2004).

Tendo em conta o exposto, objetivou-se com esse trabalho verificar o perfil nutricional e consumo alimentar de pacientes adultos, de ambos os gêneros, com excesso de peso, atendidos pelo Ambulatório de Nutrição da Universidade Estadual do Centro-Oeste (UNICENTRO), no município de Guarapuava - PR.

\section{Materiais e métodos}

Trata-se de uma pesquisa de caráter retrospectivo, envolvendo o levantamento de dados secundários, originários de prontuários do Ambulatório de Nutrição da Universidade Estadual do Centro-Oeste (UNICENTRO), campus CEDETEG, situada na cidade de Guarapuava, PR. Fizeram parte da amostra pacientes adultos de ambos os gêneros, que foram atendidos no Ambulatório de Nutrição no período de março de 2007 a dezembro de 2009 e que compareceram a pelo menos duas consultas de acompanhamento. Os dados foram coletados no período de junho a agosto de 2010, após aprovação do projeto pelo Comitê de Ética em Pesquisa com Seres Humanos (COMEP) da UNICENTRO, sob o registro $n^{0} 321 / 10$ - COMEP/ UNICENTRO.

As variáveis antropométricas peso, altura, circunferência da cintura $(\mathrm{CC})$, circunferência do braço (CB), bem como os dados de idade, diagnóstico clínico e nutricional, cálculos de ingestão de macronutrientes, micronutrientes e energia, gasto energético total (GET), taxa metabólica basal (TMB), condições socioeconômicas, história de ganho de peso e tratamentos já realizados para sobrepeso/obesidade foram coletadas por meio dos registros nos prontuários de cada paciente, de acordo com a anamnese nutricional padronizada pelo Ambulatório, e armazenadas em um banco de dados do Software Microsoft Excel ${ }^{\circledR}$.

Avaliou-se a perda de peso (\%PP) e perda de excesso de peso (\%PEP) dos pacientes sobrepeso/ obesos adultos, utilizando as seguintes fórmulas, respectivamente: $\% \mathrm{PP}=[($ peso usual - peso atual $) /$ peso usual $]$ x 100 e $\% \mathrm{PEP}=[($ peso usual - peso atual $) /$ (peso usual - peso ideal)] x 100. O Índice de Massa
Corporal (IMC) foi obtido pela multiplicação do peso em quilos pela altura em metros, elevada ao quadrado, e classificado conforme o proposto pela Organização Mundial de Saúde - OMS (1997). O IMC de $25 \mathrm{~kg} / \mathrm{m}^{2}$ foi utilizado como menor limite de excesso de peso, e assim foi possível calcular o percentual de perda de IMC (\%PIMC). (ORIA e MOOREHEAD, 2009). O peso ideal foi definido tendo como base um IMC comum, sendo este de $22,5 \mathrm{~kg} / \mathrm{m}^{2}$ para homens e de $21,5 \mathrm{~kg} / \mathrm{m}^{2}$ para mulheres. (QUADROS et al., 2007). Considerou-se peso usual aquele que o paciente apresentou na primeira consulta.

A ingestão de macronutrientes (carboidratos, fibra dietética, proteínas e lipídios) e micronutrientes (vitamina $\mathrm{C}$, vitamina $\mathrm{A}$, ferro, cálcio, sódio) foi avaliada através de inquéritos dietéticos, do tipo Recordatório de 24 horas, contidos no prontuário dos pacientes. Os resultados obtidos da avaliação de macro e micronutrientes foram analisados comparativamente às recomendações propostas pelas Dietary Reference Intakes - DRIs (2004) de acordo com idade e gênero. $\mathrm{O}$ valor da ingestão calórica foi comparado com o GET obtido pela fórmula da DRIs para gênero, idade e atividade física.

Comparou-se a ingestão de calorias e nutrientes encontrada no primeiro R24h, com a ingestão prescrita no plano alimentar e a ingestão relatada no último R24h, para avaliar a adequação da ingestão diária e verificar a adesão ao plano alimentar.

A análise dos dados se deu por meio de frequências, médias e desvio padrão. Já a análise estatística foi realizada por meio do teste $\mathrm{T}$ e Coeficiente de correlação Pearson com auxílio do Software SPSS ${ }^{\circledR}$.

\section{Resultados}

Foram analisados 22 prontuários de pacientes adultos, de ambos os gêneros, sendo $72,7 \%(\mathrm{n}=16)$ do gênero feminino. A idade média encontrada foi $40,5 \pm 8,3$ anos. Constatou-se que todos os pacientes possuíam acesso a água encanada, coleta de lixo e energia elétrica, sendo que $13,6 \%(n=3)$ não possuíam acesso a esgoto encanado. Em relação à moradia, $77,3 \%(n=17)$ relataram morar em casa de alvenaria, $4,5 \%(n=1)$ em casa de madeira e $18,2 \%(n=4)$ em casa mista, sendo em média $3,7 \pm 1,06$ pessoas por casa, variando de 2 a 6 pessoas, com renda média familiar de $\mathrm{R} \$ 2.024,8 \pm 1.308,58$ e renda per capita de $\mathrm{R} \$ 599,2 \pm 460,15$. 
No quadro 1 encontram-se descritas as informações a respeito da escolaridade e ocupação dos pacientes avaliados, em percentual (\%) e número (n). Pode-se verificar que se tratou de uma amostra heterogênea, com distintos níveis de escolaridade e ocupação, sendo que a maior parte dos indivíduos relatou trabalhar no lar e possuir ensino médio completo ou incompleto.

Quadro 1- Escolaridade e ocupação dos pacientes atendidos pelo Ambulatório de Nutrição

\begin{tabular}{|l|c|l|c|}
\hline Escolaridade & \% (n) & \multicolumn{1}{|c|}{ Ocupação } & \% (n) \\
\hline Pós-graduação & $9,1(2)$ & Do lar & $22,7(5)$ \\
\hline $\begin{array}{l}\text { Ensino superior } \\
\text { incompleto }\end{array}$ & $9,1(2)$ & $\begin{array}{l}\text { Auxiliar } \\
\text { administrativo }\end{array}$ & $9,1(2)$ \\
\hline $\begin{array}{l}\text { Ensino superior } \\
\text { completo }\end{array}$ & $18,2(4)$ & $\begin{array}{l}\text { Técnico em } \\
\text { enfermagem }\end{array}$ & $4,5(1)$ \\
\hline $\begin{array}{l}\text { Ensino médio } \\
\text { incompleto }\end{array}$ & $18,2(4)$ & Motorista & $4,5(1)$ \\
\hline $\begin{array}{l}\text { Ensino médio } \\
\text { completo }\end{array}$ & $18,2(4)$ & Vendedora & $4,5(1)$ \\
\hline $\begin{array}{l}\text { Ensino } \\
\text { fundamental } \\
\text { completo }\end{array}$ & $13,6(3)$ & Mecânico & $4,5(1)$ \\
\hline $\begin{array}{l}\text { Ensino } \\
\text { fundamental } \\
\text { incompleto }\end{array}$ & $13,6(3)$ & Empresário & $4,5(1)$ \\
\hline & Assistente de cartório & $4,5(1)$ \\
\hline & Manicure & $4,5(1)$ \\
\hline & Assistente social & $4,5(1)$ \\
\hline Ça do trabalho & $4,5(1)$ \\
\hline & Professor & $4,5(1)$ \\
\hline & Cabeleireira & $4,5(1)$ \\
\hline
\end{tabular}

A tabela 1 apresenta dados comparativos entre os valores de CC, CB, IMC, TMB, GET e peso na primeira e última consulta nutricional. Traz ainda \%PP, \%PEP e \%PIMC até a última consulta nutricional, além das médias de estatura e de peso ideal (PI).

Nota-se que não houve diferença estatística significativa entre o IMC inicial e final, peso inicial e final, CB inicial e final, e entre CC inicial e final ( $p>0,05)$, através do Teste $T$, indicando que não ocorreram mudanças significativas entre as medidas obtidas na primeira consulta e no último acom- panhamento nutricional. Porém, há uma correlação moderadamente positiva entre o IMC inicial e CC inicial $(\mathrm{r}=0,73)$, indicando que os valores de $\mathrm{CC}$ modificam-se de acordo com o IMC. Em relação ao peso, também não foi observada diferença estatística significativa, com relação ao peso inicial e final, podendo-se observar ainda que, em média, os pacientes apresentavam aproximadamente $30 \mathrm{~kg}$ a mais que o PI médio.

Tabela 1- Perfil antropométrico e necessidade energética inicial e final de pacientes atendidos no Ambulatório de Nutrição.

\begin{tabular}{l|l|l|c}
\hline Variável & \multicolumn{1}{|c|}{ Inicial } & \multicolumn{1}{c}{ Final } & p* \\
\hline CC & $97,6 \pm 11,4$ & $96,5 \pm 10,8$ & 0,89 \\
\hline CB & $34,2 \pm 4,2$ & $32,2 \pm 4,9$ & 0,09 \\
\hline Peso & $84,5 \pm 14,3$ & $82,8 \pm 14,9$ & 0,35 \\
\hline IMC & $32,4 \pm 5,7$ & $31,7 \pm 5,6$ & 0,68 \\
\hline TMB & $1.581,8 \pm 195,5$ & $1.566,7 \pm 203,1$ & 0,40 \\
\hline GET & $2.373,1 \pm 315,1$ & $2.385,7 \pm 292,7$ & 0,45 \\
\hline & Média & Desvio padrão & \\
\hline \%PP & 2,1 & 4,0 & \\
\hline \%PEP & 6,8 & 15,3 & \\
\hline \%PIMC & $-6,7$ & 133,2 & \\
\hline Estatura & 1,6 & 0,1 & \\
\hline PI & 57,2 & 7,3 & \\
\hline
\end{tabular}

*Valores obtidos pela comparação dos dados por meio do teste $\mathrm{T}$ de Student.

Entre as doenças associadas ou não à obesidade, que podem ter seus efeitos controlados com a redução do peso, verificou-se a incidência de câncer de mama, varizes, HAS, hiperuricemia, hipotireoidismo, esteatose hepática, espondilose cervical, fibromialgia, artrose, gastrite, hipercolesterolemia, baixos níveis de HDL-colesterol, DM, hipertrigliceridemia e níveis elevados de LDL-colesterol, entre os indivíduos da amostra.

O histórico de ganho de peso dos pacientes também foi avaliado com base nos dados obtidos na anamnese nutricional. Observou-se que o peso máximo atingido, em média, foi de $87,3 \pm 14,4$ quilos, com idade média de $36,1 \pm 10,3$ anos, e o peso mínimo foi de $62,9 \pm 10,1$ quilos, com 23,2 $\pm 8,9$ anos. Dos pacientes analisados, $40,9 \%(\mathrm{n}=9)$ relataram que já haviam realizado algum tipo de tratamento para obesidade, 

POR UM AMBULATÓRIO DE NUTRIÇÃO

através do uso de medicamentos: $13,6 \%(\mathrm{n}=3)$, dietas da moda: $27,3 \%(\mathrm{n}=6)$, acompanhamento com médico: $9,1 \%(\mathrm{n}=2)$, e nutricionista: $27,3 \%(\mathrm{n}=6)$, estando estes associados ou não.

Na tabela 2 pode-se analisar os valores de energia, macronutrientes, proteína por quilo de peso (Ptn $(\mathrm{g}) / \mathrm{kg})$ e micronutrientes relatados na primeira consulta e último acompanhamento, verificados por meio do inquérito de consumo alimentar do tipo R24h, expressos em quilocalorias (kcal), percentual (\%), grama $(\mathrm{g})$, miligrama $(\mathrm{mg})$ e micrograma $(\mathrm{mcg})$. Além disso, pode-se comparar a ingestão alimentar anterior e posterior à intervenção nutricional. Os valores de $\mathrm{p}$ indicam a comparação entre R24h inicial e final e R24h final com o cardápio prescrito pelos acadêmicos do Curso de Nutrição.

Tabela 2- Comparação da ingestão alimentar e energética anterior e posterior à intervenção nutricional, e diferença com o plano alimentar calculado.

\begin{tabular}{l|c|c|c|c|c}
\hline \multicolumn{1}{c|}{ Variáveis } & R24 h Inicial & R24 h Final & p & Plano Alimentar & P \\
\hline Energia (kcal) & $1.663,7 \pm 592,6$ & $1.400 \pm 529,9$ & 0,06 & $1.893,7 \pm 300$ & $0,0002 *$ \\
\hline Carboidrato (\%) & $52,1 \pm 8,2$ & $57,6 \pm 8,7$ & $0,02 *$ & $58,1 \pm 3,8$ & 0,417 \\
\hline Proteína (\%) & $15,9 \pm 4,5$ & $17,4 \pm 5,7$ & 0,2 & $19,9 \pm 3,8$ & $0,045^{*}$ \\
\hline Ptn (g)/ kg & $0,7 \pm 0,2$ & $0,7 \pm 0,3$ & 0,4 & $1,3 \pm 0,8$ & $0,003 *$ \\
\hline Lipídio (\%) & $31,6 \pm 9,1$ & $24,8 \pm 7,1$ & $0,004 *$ & $22 \pm 3,1$ & $0,048^{*}$ \\
\hline Fibra dietética (g) & $13,7 \pm 7,7$ & $14,3 \pm 7,2$ & 0,4 & $26,5 \pm 8,4$ & $0,00001 *$ \\
\hline Sódio (mg) & $1.394,3 \pm 837,3$ & $1.029,7 \pm 632,7$ & 0,1 & $960,2 \pm 719,1$ & 0,38 \\
\hline Cálcio (mg) & $472,7 \pm 207,2$ & $524,4 \pm 306,2$ & 0,3 & $936,6 \pm 337,7$ & $0,0001 *$ \\
\hline Ferro (mg) & $10,3 \pm 4,9$ & $9,5 \pm 5,0$ & 0,3 & $15,3 \pm 3,6$ & $0,00003^{*}$ \\
\hline Vitamina A (mcg) & $271,7 \pm 188,7$ & $512,2 \pm 464,3$ & $0,015^{*}$ & $1.586,3 \pm 943,3$ & $0,00001^{*}$ \\
\hline Vitamina C (mg) & $49,6 \pm 59$ & $82,6 \pm 87,9$ & 0,1 & $164,6 \pm 88,6$ & $0,002^{*}$ \\
\hline
\end{tabular}

"Na comparação entre os grupos os dados são estatisticamente diferentes pelo teste T de Student.

Encontrou-se ingestão abaixo do preconizado para vitamina $\mathrm{C}$, tanto para homens quanto para mulheres, no primeiro R24h, com melhora sem significância estatística no último R24h, mas com grande variação de consumo entre os pacientes, conforme pode ser visto pelo desvio padrão. Em relação ao consumo de ferro observa-se que não houve modificações significativas na primeira e última consulta de acompanhamento, e que a média é suficiente para o gênero masculino e insuficiente para o feminino, mas destaca-se a discrepância nos valores de desvio padrão.

O consumo de cálcio e fibra dietética encontra-se abaixo do recomendado nos dois R24h. Já a ingestão de vitamina A apresentou diferença estatística significante do primeiro R24h para o último, mostrando aumento na ingestão, mas ainda sem atingir os níveis preconizados nas DRIs. Os macronutrientes e sódio enquadram-se nos limites estabelecidos como adequados, com atenção à ingestão média inferior ao ideal de proteína por quilo por dia. No entanto, a média de consumo energético diminuiu do primeiro para o último R24h, não atingindo o prescrito no plano alimentar e, ainda, com média inferior à TMB no R24 final. Porém, deve-se atentar para a grande variação do desvio padrão.

\section{Discussão}

Identificou-se que há maior procura por atendimento nutricional pelo gênero feminino, com altos níveis de escolaridade, renda e condições básicas de moradia, mostrando que essa população parece ter mais acesso à informação e maior preocupação com questões de saúde e estética, levando esses indivíduos a procurar aconselhamento nutricional. No entanto, pode-se verificar o mesmo nos pacientes com menor nível de instrução, parecendo este fator não interferir no surgimento do sobrepeso/obesidade no presente estudo. Segundo Pesquisa Nacional sobre Saúde e Nutrição, realizada em 1989 no Brasil, o grau de 
instrução parece ter sido determinante para o controle da obesidade, apesar de os índices ainda permanecerem altos. Este fato mostra as mudanças ocorridas daquele período até o atual, destacando-se a transição nutricional devido às modificações demográficas e epidemiológicas. (FILHO, MIGLIOLI, SANTOS, 2007; GIGANTE et al., 2008).

A partir da análise dos dados antropométricos, observaram-se valores elevados para $\mathrm{CC}$ de acordo com o preconizado pela Sociedade Brasileira de Cardiologia (2007) e IMC, tanto para homens quanto para mulheres, constituindo-se estes em fatores de risco para doenças cardiovasculares e metabólicas. Em estudo de Perozzo et al. (2008), do tipo transversal de base populacional que avaliou 1.026 mulheres da região Sul, encontrou-se maior prevalência de obesidade abdominal, com $23,3 \%$ das mulheres apresentando $\mathrm{CC}=88 \mathrm{~cm}$ (nível II) e prevalência de $18 \%$ de mulheres obesas $\left(\mathrm{IMC}=30 \mathrm{~kg} / \mathrm{m}^{2}\right.$ ). Dados esses preocupantes para a amostra estudada e população em geral, pois a Síndrome Metabólica (SM) envolve um conjunto de no mínimo três fatores de risco, destacando-se a HAS, hipertrigliceridemia, hipercolesterolemia, diminuição do HDL-colesterol e tolerância à glicose diminuída/DM tipo 2, todos encontrados em nossa pesquisa, usualmente relacionados à deposição central de gordura, obesidade e resistência à insulina, responsável pelo aumento da mortalidade nessa faixa etária por doenças cardiovasculares (KEARNEY e MCELHONE, 1999; PEROZZO et al., 2008; OLIVEIRA et al., 2009).

Não houve alteração significativa na TMB e GET, assim como nos resultados de perda ponderal de peso e IMC (tabela 1) dos indivíduos estudados, sugerindo pequena adesão ao plano alimentar proposto, principalmente quando se observa o consumo energético menor que o proposto pelo plano alimen$\operatorname{tar}$ (tabela 2).

Corroborando os resultados observados no presente trabalho, a pesquisa de Guimarães et al. (2010), relacionada ao padrão alimentar quantitativo de obesos, encontrou redução média não significativa estatisticamente no consumo energético em relação a situações anteriores e posteriores em três meses de acompanhamento. Este resultado pode ser justificado pelo fato de indivíduos obesos apresentarem inabilidade em avaliar adequadamente o padrão alimentar, podendo subestimar o valor energético da dieta ha- bitual, principalmente por considerarem seu padrão de ingestão suficientemente saudável. (KEARNEY e MCELHONE, 1999).

Sabe-se que o consumo de dietas restritivas em termos energéticos altera os mecanismos do organismo para armazenamento de energia, remetendo-o a épocas primitivas em que havia elevada restrição de alimentos. Em consequência, o corpo armazena energia mesmo com o consumo de dietas hipocalóricas (termogênese adaptativa). Justifica-se, assim, a prescrição dietética visando modificar esse mecanismo, com consequente promoção de perda de peso. Se não fosse este fato, os resultados de ingestão energética encontrados não explicariam o excesso de massa corporal, mesmo os valores de GET sendo maiores nos indivíduos obesos. Isso sugere que o organismo é programado para proteger-se mais intensamente contra a perda do que contra o ganho de peso corporal, dificultando a perda e manutenção de peso adequado em indivíduos obesos, tornando-se preocupante a diminuição do GET nos períodos de restrição alimentar, que possivelmente se mantém mesmo após o retorno da ingestão energética habitual. (FANTINO e CABANAC, 1980; LEVINE, 2005; MELO, TIRAPEGUI e RIBEIRO, 2008; SOUZA et al., 2010).

A dieta é uma forma de tratamento que restringe a ingestão energética total para causar um balanço negativo e consequentemente a perda de peso. Uma restrição rígida pode trazer resultados em curto prazo, mas não em longo prazo. Dietas com restrições muito severas são indesejáveis e perigosas para a saúde, por originarem grandes perdas de água, eletrólitos, minerais, glicogênio e outros tecidos isentos de gordura, num processo associado a uma mínima redução de tecido adiposo. (LOTTENBERG, 2006; CASAZZA et al., 2013).

A obesidade necessita de tratamento de longo prazo para a obtenção de resultados satisfatórios com relação à perda e manutenção de peso corporal. Para cada $10 \%$ de aumento no peso corporal, geralmente causado por excessos alimentares e redução na prática de atividade física, há aumento na incidência de doenças coronarianas em aproximadamente $20 \%$, devido aos fatores de risco associados à obesidade e SM. No entanto, perda ponderal modesta (5\% a $10 \%)$ resulta na melhora de diversos parâmetros clínicos, mesmo que o indivíduo ainda permaneça com o IMC superior a $30 \mathrm{~kg} / \mathrm{m}^{2}$, como redução da pressão arte- 
rial e níveis glicêmicos. (WILLIANSON, PAMUK e THUN, 1995; SCHEEN e LEFÈBVRE, 1999; FRANCISCHI et al., 2000; LOTTENBERG, 2006).

Verificou-se que o peso máximo atingido pela amostra foi atingido na idade adulta, podendo estar relacionado a vários fatores, considerando que a obesidade caracteriza-se como uma doença crônica e multifatorial, com causas ambientais, nutricionais e genéticas. (BRASIL, 2007). Com isso, houve a tentativa de vários tratamentos, todos, até o presente momento, ineficazes. Fato este que pode ter várias explicações, entre elas a busca pelo emagrecimento rápido, fazendo surgir a procura por dietas alternativas denominadas "dietas da moda" e utilização de medicamentos, muitas vezes sem acompanhamento especializado. (LOTTENBERG, 2006).

Geralmente essas dietas oferecem produtos substitutos das refeições, de baixa caloria e indicados para substituir uma ou duas refeições por dia. O ideal é que não sejam utilizados como única fonte de nutrição e que haja a supervisão de um especialista, porém isto não é observado no cotidiano. Além disso, não devem ser usadas por períodos prolongados, pois são formulações que permitem a perda de peso, mas não sua manutenção. (COPPINI e WAITZBERG, 2006).

Avaliando-se o consumo alimentar na primeira e última consulta notou-se melhora na ingestão de alguns nutrientes e sua distribuição percentual, como lipídios e carboidratos, porém todos os macronutrientes (carboidratos, proteínas e lipídios), em ambos os períodos, apresentaram distribuição percentual dentro dos limites padronizados pelo Instituto de Medicina (2004). Um estudo de Nordmann et al. (2006) demonstrou que, no período de um ano, uma dieta com baixo conteúdo de gordura, além de promover melhores efeitos no perfil lipídico, foi mais eficaz na redução de peso do que as dietas com baixo teor de carboidratos.

O presente trabalho verificou ainda um aumento significativo no consumo de carboidratos na primeira consulta em relação à última, podendo ter refletido nos índices de ingestão de lipídios, promovendo mudanças benéficas que foram introduzidas após recebimento do plano alimentar e orientações nutricionais.

Destacam-se também os baixos índices de fibra dietética consumidos pela população em questão, em relação às 25 gramas diárias recomendadas pela $\mathrm{FAO} /$
OMS (2003). A fibra dietética é um grande polímero de carboidrato não hidrolisado por enzimas endógenas no intestino humano, importante para aumentar o volume fecal, prevenir diversos tipos de câncer e melhorar o funcionamento intestinal. Além disso, resultados de estudos epidemiológicos sugerem que os carboidratos com grande quantidade de fibras e baixo índice glicêmico ou carga glicêmica diminuem o risco de diabetes mellitus, previnem obesidade e ganho de peso. (MELLO e LAAKSONEN, 2009). Daí a importância do estímulo ao consumo de frutas, vegetais e grãos integrais durante $\mathrm{o}$ aconselhamento nutricional.

A ingestão de proteína por quilo de peso por dia mostrou-se abaixo do recomendado pela Recommended Dietary Allowances (RD) de 1989, de 0,8g/ $\mathrm{kg} / \mathrm{dia}$, indicando o consumo de uma dieta hipoproteica mesmo após o aconselhamento nutricional e distribuição percentual adequada. Resultado diferente do encontrado por SOUZA et al. (2010) em população adulta de Niterói (RJ), que apresentou ingestão de proteína por quilo por dia levemente superior à recomendada $(1,28 \pm 0,03)$.

A ingestão adequada de vitaminas e minerais é importante para a manutenção das diversas funções metabólicas do organismo, sendo que a deficiência pode produzir manifestações patológicas (VELÁSQUEZ-MELÉNDEZ et al., 1997), como, por exemplo, a baixa ingestão de ferro associada a níveis baixos de consumo de vitamina $C$, que é responsável pelo aumento da absorção do ferro dietético, podendo estar associada à ocorrência de anemia, alteração da resposta imune, redução da capacidade de trabalho e desempenho intelectual, conforme também foi verificado em estudo de Lopes et al (2005). Além disso, a vitamina $\mathrm{C}$ atua como cofator ou cossubstrato de diferentes enzimas, envolvida na biossíntese de colágeno, ATP, norepinefrina e no metabolismo da tirosina. Também possui ação na conversão do colesterol em ácidos biliares, no metabolismo iônico de minerais e no processo de prevenção de doenças crônicas não transmissíveis, no sistema imunológico e no processo de cicatrização. (COZZOLINO, 2007).

O consumo de sódio mostrou-se adequado nos dois períodos avaliados, sugerindo informação dos pacientes em relação aos malefícios de uma dieta rica neste mineral e controle dietéticos dos indivíduos que já relataram ser hipertensos. Além disso, este mineral é importante para a transmissão dos impulsos 
nervosos e ação muscular. Os baixos índices de consumo de cálcio e vitamina A tornam-se prejudiciais, já que o cálcio é fundamental para a prevenção de osteoporose e para a manutenção da pressão arterial, principalmente pelo fato da amostra ser composta basicamente por mulheres, e pela vitamina $\mathrm{A}$ iniciar o impulso nervoso, agir como carreador de resíduos de manosil, na síntese de glicoproteínas, na produção de muco e na resistência a infecções, mediada pela ação moduladora da resposta imune. (COZZOLINO, 2007).

Os achados da nossa pesquisa assemelham-se aos do estudo de Fisberg et al. (2013), que avaliou a ingestão de micronutrientes na população idosa, e aos da pesquisa de Araujo et al. (2013), que avaliou micronutrientes em adultos. Em relação ao sódio, ambos os estudos encontraram níveis acima dos valores recomendados, diferenciando-se dos dados obtidos no Ambulatório de Nutrição. Porém, vale ressaltar que a ingestão de sódio é de difícil mensuração, podendo o individuo hiper ou subestimar sua ingestão, principalmente tratando-se da quantidade utilizada para o preparo das refeições, além da mesma ser diferenciada do encontrado nas tabelas padrões de composição nutricional dos alimentos. Por basear-se nessas tabelas, estima-se a ingestão de nutrientes, não conseguindo obter-se uma avaliação precisa, visto que o alimento está suscetível a variações de solo e clima, por exemplo, que influenciarão em seu conteúdo de micronutrientes.

Os achados no presente trabalho, em relação aos R24h, evidenciaram dificuldades na adesão ao plano alimentar e no alcance de adequada ingestão de nutrientes, podendo comprometer a saúde dos indivíduos. Inelmen et al. (2005) apontam que problemas pessoais, ausência de apoio familiar, falta de motivação e os resultados negativos na perda de peso durante o tratamento são fatores importantes na taxa de desistência do tratamento nutricional, e que estudos clínicos têm demonstrado taxa de desistência de até $80 \%$. Estudo que avaliou a adesão a dois métodos de aconselhamento nutricional encontrou taxa de desistência superior a $50 \%$ entre os participantes de cada grupo. (GUIMARÃES et al., 2010).

Segundo Rodrigues et al. (2005), o paciente precisa tornar-se capaz de identificar barreiras para o autocontrole e desenvolver mecanismos para superá-las, para deste modo obter melhores resultados no tratamento da obesidade, prevenindo recaídas e adquirindo habilidade para resolver problemas cotidianos. Através da educação nutricional aliada à prática clínica, pode-se trabalhar o descrito anteriormente, o que também irá auxiliar na concretização do acesso e incorporação de hábitos de vida saudáveis que promovam a saúde do indivíduo, por estimular e fornecer conhecimento para escolhas saudáveis no cotidiano. (MIRANDA e GOMES, 2007).

Como o presente estudo consistiu numa pesquisa retrospectiva, baseada em análise de arquivos, houve necessidade de exclusão de vários prontuários da amostra devido à ausência de dados, sendo esta a maior dificuldade enfrentada para a realização da mesma. Em vista disto, salienta-se a importância do correto preenchimento da anamnese nutricional e dos cálculos dos inquéritos dietéticos, para que se possa acompanhar e analisar a população que frequenta o Ambulatório, auxiliando no delineamento de medidas educativas e comportamentais para eficácia do tratamento nutricional no local, também servindo como guia para outros centros de atendimento nutricional.

\section{Considerações finais}

Evidenciou-se pequena adesão, por parte dos pacientes, ao tratamento nutricional, tanto pelos parâmetros antropométricos quanto pela ingestão alimentar após aconselhamento nutricional. A maior parte da amostra estudada foi composta por mulheres, fato que pode estar relacionado com a preocupação em relação à estética, sem interferência do nível social.

Não houve redução significativa entre as medidas de peso, IMC e/ou de medidas antropométricas na primeira consulta e último acompanhamento. Esses achados são confirmados pelo não seguimento do plano alimentar prescrito, observando-se baixa ingestão energética e de micronutrientes pela população em questão, fato que pode levar ao desenvolvimento de doenças carenciais, bem como a modificações metabólicas que prejudicam o processo de emagrecimento e saúde do indivíduo. Porém, observou-se melhora na distribuição percentual dos macronutrientes, principalmente de lipídios, o que pode promover benefícios em nível hematológico na dosagem sérica de colesterol, triglicerídeos, entre outros. Dessa forma, verificou-se que mesmo uma redução mínima de peso pode conseguir controlar e/ou reduzir os efeitos das doenças associadas à obesidade. 

POR UM AMBULATÓRIO DE NUTRIÇÃO

Sendo assim, fica clara a necessidade da educação nutricional após observação dos hábitos alimentares errôneos de cada população, a fim de auxiliar os indivíduos no encorajamento para enfrentar os obstáculos do cotidiano, bem como na adequada escolha e incorporação de novos hábitos e estilos de vida em prol da saúde.

\section{Referências}

ARAUJO, M. C. et al. Consumo de macronutrientes e ingestão inadequada de micronutrientes em adultos. Revista de Saúde Pública. v.47, n. 1, p. 177S-89S, 2013.

ASSIS, M. A.; NAHAS, M. V. Aspectos motivacionais em programas de mudança de comportamento alimentar. Revista Nutrição de Campinas. v. 12, n. 1, p. 33-41, 1999.

AVENELL, A. et al. What are the long-term benefits of weight reducing diets in adults? A systematic review of randomized controlled trials. Journal of Human Nutrition and Dietetics. v. 17, n. 4, p. 317-35, 2004.

BRASIL. Instituto Nacional de Alimentação e Nutrição. Pesquisa Nacional sobre Saúde e Nutrição (PNSN) - 1989. Arquivo da Pesquisa. Brasília, 1990.

Ministério da Saúde. Secretaria-Executiva. Secretaria de Atenção Básica. Glossário temático: alimentação e nutrição. Brasília: Editora do Ministério da Saúde, 2007.

CASAZZA, K. et al. Myths, presumptions, and facts about obesity. New England Journal of Medicine. v. 368, n. 5, p. $446-454,2013$

COPPINI, L. Z.; WAITZBERG, D. L. Obesidade: Abordagem dietética. In: WAITZBERG, D. L. Nutrição oral, enteral e parenteral na prática clínica. 3. ed. 2 vol. São Paulo: Atheneu, 2006.

COZZOLINO, S. M. F. Biodisponibilidade de nutrientes. 2. ed. Barueri, SP: Manole, 2007.

FANTINO, M.; CABANAC, M. Body weight regulation with a proportional hoarding response in rat. Physiology \& Behavior. v. 24, n. 5, p. 934-42, 1980.

FEIGEBAUM, A. et al. Influence of intense multidisciplinary follow-up and orlistat on weight reduction in a primary care setting. BMC Family Practice. v. 1, p. 1-7, 2005.

FILHO, M. B.; Miglioli, T. C.; SANTOS, M. C. Anthropometric normality in adults: the geographical and socio-economic paradox of the nutritional transition in Brazil. Revista Brasileira de Saúde Materno Infantil. v. 7, n. 4, p. 487-493, 2007.

FISBERG, R. M. et al. Ingestão inadequada de nutrientes na população de idosos do Brasil: Inquérito Nacional de Alimentação 2008-2009. Revista de Saúde Pública. v.47, n. 1, p. 222S-30S, 2013.
FONTAINE, K. R. et al. Years of life lost due to obesity. Journal of the American Medical Association (JAMA). v. 289, p. 187-93, 2003.

FRANCISCHI, R. P. P. et al. Obesidade: atualização sobre sua etiologia, morbidade e tratamento. Revista Nutrição, Campinas, SP. v. 3, n. 1, p. 17-28, 2000.

GIGANTE, D. P. et al. Avaliação nutricional de adultos da coorte de nascimentos de 1982, Pelotas, RS. Revista de Saúde Pública. v. 42, n.2, p. 60-9, 2008.

GUIMARÃES, N. G. et al. Adesão a um programa de aconselhamento nutricional para adultos com excesso de peso e comorbidades. Revista Nutrição. Campinas, SP. v. 23, n. 3, p. 323-333, 2010.

HARPER, A.; PETERSON, M. Viewpoint weight loss strategies: a change of focus is required. Obesity Reviews. v. 4, n.4, p. 239-40, 2004.

INELMEN, E. M. et al. Predictors of drop-out in overweight and obese outpatients. International Journal of Obesity. v. 29, n. 1, p. 122-8, 2005.

INSTITUTE OF MEDICINE, FOOD AND NUTRITION BOARD. Dietary reference intakes. Vitamins, elements, energy and macronutrients. Washington: National Academy of Sciences, 2004.

KEARNEY, J. M.; MCELHONE, S. Perceived barriers in trying to eat healthier: results of a pan-EU consumer attitudinal survey. British Journal of Nutrition. v. 81, n. 2, p. 133 S-7S, 1999.

LEVINE, J. A. Measurement of energy expenditure. Public Health Nutrition. v.8, n. 7A, p. 1123-32, 2005.

LINDTRÖM , J. et al. Long-term effects and economic consequences of treatments for obesity and implications for health improvement. Health Technology Assessment. v. 8, n. 21, p. 1-182, 2004.

LOPES, A. C. S. et al. Consumo de nutrientes em adultos e idosos em estudo de base populacional: Projeto Bambuí. Cadernos de Saúde Pública. v.21, n.4, p. 1201-1209, 2005.

LOTTENBERG, A. M. P. Tratamento dietético da obesidade. Einstein. v. 1, p. S23-S28, 2006.

MELLO, V. D.; LAAKSONEN, D. E. Fibras na dieta: tendências atuais e benefícios à saúde na síndrome metabólica e no diabetes melito tipo 2. Arquivo Brasileiro de Endocrinologia e Metabologia. v. 53, n. 5, 2009.

MELO, C. M.; TIRAPEGUI, J.; RIBEIRO, S. M. L. Gasto energético corporal: conceitos, formas de avaliação e sua relação com a obesidade. Arquivo Brasileiro de Endocrinologia e Metabologia. v. 52, n. 3, 2008.

MIRANDA, J.; GOMES, P. T. T. Educação nutricional e sua importância no combate dos quadros alarmantes de obesidade. Revista Eletrônica Lato Sensu. v. 2, n. 1, 2007. 
MONTEIRO, C. A. Velhos e novos males da saúde no Brasil. 2 ed. São Paulo: Hucitec, 2000.

NATIONAL ACADEMY PRESS. Recommended Dietary Allowances. 10. ed. Washington, 1989.

NORDMANN, A. J. et al. Effects of low-carbohydrate vs. low fat diets on weight loss and cardiovascular risk factors. A meta-analysis of randomized controlled trials. Archives of Internal Medicine. v. 166, p. 285-93, 2006.

OLIVEIRA, R. M. S. et al. Influência do estado nutricional pregresso sobre o desenvolvimento da síndrome metabólica em adultos. Arquivo Brasileiro de Cardiologia. v. 92, n. 2, p. 107-112, 2009.

ORIA, H. E.; MOOREHEAD, M. K. Updated Bariatric Analysis and Reporting Outcome System (BAROS). Surgery for obesity and related disease. v. 5, p. 60-66, 2009.

PEROZZO, G. et al. Associação dos padrões alimentares com obesidade geral e abdominal em mulheres residentes no Sul do Brasil. Cadernos de Saúde Pública. v. 24, n. 10, p. 2427-2439, 2008.

PRICE, S. Understanding the importance to health of a balances diet. Nurses Times. v. 101, n. 1, p. 30-31, 2005.

QUADROS, M. R. R. et al. Intolerância alimentar no pósoperatório de pacientes submetidos à cirurgia bariátrica. Revista Brasileira de Nutrição Clínica. v. 22, n. 1, p. 159, 2007.

RODRIGUES, E. M. et al. Resgate do conflito de aconselhamento no contexto do atendimento nutricional. Revista de Nutrição. v.18, n. 1, p. 119-28, 2005.

SCHEEN, A. J.; LEFÈBVRE, P. J. Management of the obese diabetic patient. Diabetes Reviews. v.7, p. 77-93, 1999.

SOCIEDADE BRASILEIRA DE CARDIOLOGIA - SBC. IV Diretriz Brasileira Sobre Dislipidemias e Prevenção da Aterosclerose. Departamento de Aterosclerose da Sociedade Brasileira de Cardiologia. IV Diretriz da Sociedade Brasileira de Cardiologia. Arquivo Brasileiro de Cardiologia. v. 88, n. 1, 2007.

SOUZA, D. R. et al. Ingestão alimentar e balanço energético da população adulta de Niterói, Rio de Janeiro, Brasil: resultados da Pesquisa de Nutrição, Atividade Física e Saúde (PNAFS). Cadernos de Saúde Pública. v. 26, n.5, p. 879-890, 2010.

SOUZA, L. J. et al. Prevalência de obesidade e fatores de risco cardiovascular em Campos, Rio de Janeiro. Arquivo Brasileiro de Endocrinologia e Metabologia. v. 47, p. 669-76, 2003.

VELÁSQUEZ-MELÉNDEZ, G. et al. Consumo alimentar de vitaminas e minerais em adultos residentes em área metropolitana de São Paulo, Brasil. Revista de Saúde Pública. v. 31, n. 2, 1997.
WILLIANSON, D. F.; PAMUK, E.; THUN, M. Prospective study of intentional weight loss and mortality in neversmoking over weight US white women aged 40-64 years. American Journal of Epidemiology. v. 141, p. 1128-41, 1995.

WORLD HEALTH ORGANIZATION - WHO. Obesity: Preventing and Managing the Global Epidemic. Report of a WHO Consultation. WHO Technical Report Series n. 894. Geneva, 2004.

Obesity: Preventing and managing the global epidemic. Geneva; 1997.

Diet, nutrition and the prevention of chronic diseases. 2003. 